\title{
TEMPERATURE DEPENDENCE OF SPIN CURRENTS CARRIED BY JORDAN-WIGNER FERMIONS AND MAGNONS IN INSULATORS
}

$\operatorname{AUTHOR}(S):$

NAKATA, KOUKI

\section{CITATION:}

NAKATA, KOUKI. TEMPERATURE DEPENDENCE OF SPIN CURRENTS CARRIED BY JORDAN-WIGNER FERMIONS AND MAGNONS IN INSULATORS. International Journal of Modern Physics B 2012, 26(01): 1250011.

\section{ISSUE DATE:}

2012

URL:

http://hdl.handle.net/2433/152378

\section{RIGHT:}

(C) 2012 World Scientific Publishing Co.; This is not the published

version. Please cite only the published version.; この論文は出版社版で ありません。引用の際には出版社版をご確認ご利用ください。 


\title{
TEMPERATURE DEPENDENCE OF SPIN CURRENTS CARRIED BY JORDAN-WIGNER FERMIONS AND MAGNONS IN INSULATORS
}

\author{
KOUKI NAKATA \\ Yukawa Institute for Theoretical Physics, \\ Kyoto University, \\ Kitashirakawa Oiwake-Cho, Kyoto 606-8502, Japan \\ nakata@yukawa.kyoto-u.ac.jp \\ Received Day Month Year \\ Revised Day Month Year
}

\begin{abstract}
The temperature dependence of spin currents in insulators at the finite temperature near zero Kelvin is theoretically studied. The spin currents are carried by Jordan-Wigner fermions and magnons in one- and three- dimensional insulators. These spin currents are generated by the external magnetic field gradient along the quantization axis and also by the two-particle interaction gradient. In one-dimensional insulators, quantum fluctuations are strong and the spin current carried by Jordan-Wigner fermions shows the stronger dependence on temperatures than the one by magnons.
\end{abstract}

Keywords: Spin currents; Jordan-Wigner fermions; Magnons; Insulators; Spintronics.

\section{Introduction}

Recently a new branch of physics and nanotechnology called spintronics ${ }^{1,2}$ has emerged and spin transport phenomena in condensed matter systems have been attracting special interests because of applications to spintronics. This research field has seen a rapid development over the last decades. ${ }^{3}$ The aim of spintronics is the control and utilization of the spin as well as charge degrees of freedom of electrons. Spintronics avoids the dissipation from Joule heating by replacing charge currents with spin currents, and therefore to clarify the properties of spin currents is an important theoretical issue from viewpoints of fundamental science and potential applications. ${ }^{4,5}$

The spin current means a flow of the spin angular momentum, in general. It also flows in insulators as well as in metals where conduction electrons carry a spin current. In insulators there is no conduction electrons, but there exists an other kind of carrier, namely, spin-waves.

Experimentally, a spin-wave spin current, a spin current carried by spin-waves has already been established as a physical quantity. Y.Kajiwara et al. ${ }^{6}$ have reported that a spin-wave spin current in an insulator can be generated and detected using 
direct and inverse spin-Hall effects. ${ }^{7}$ Moreover through the effects, it is possible to convert an electric signal in a metal into a spin-wave spin current, and vice versa. This spin-wave spin current has a novel feature that this current persists for much greater distance than the conduction electron spin current, which disappears within very short distance (see Table 1 ). This novel feature of the spin-wave spin current is expected to lead to developments beyond silicon-based technologies.

Theoretically, Meier and $\operatorname{Loss}^{8}$ have studied the magnon transport in both ferromagnetic and antiferromagnetic materials. They have found that the spin conductance is quantized in the units of order $\left(g \mu_{\mathrm{B}}\right)^{2} / h$ in the antiferromagnetic isotropic materials ( $g$; the gyromagnetic ratio, $\mu_{\mathrm{B}}$; the Bohr magneton, $h$; Planck constant). Fujimoto $^{9}$ has studied a spin Hall effect of spin-waves in frustrated magnets. A longitudinal magnetic field gradient induces a transverse spin current carried by spin wave; $J_{x}=\sigma_{x y}^{\mathrm{SHE}} \partial B / \partial y, \sigma_{x y}^{\mathrm{SHE}} \propto T^{5}$ at the low-temperature, where $J_{x}$ is the spin Hall current, $\sigma_{x y}^{\mathrm{SHE}}$ is the Hall conductivity for the spin current and $B$ is the magnetic field along the quantization axis. Katsura et al. ${ }^{10,11,12}$ have reported the intrinsic thermal Hall effect for magnons due to the anomalous velocity.

Though thus theoretical studies of spin currents in insulators have developed, it should be emphasized that, besides magnons, there exists an other kind of carrier in insulators, namely, the Jordan-Wigner ( J-W) fermion. This carrier is peculiar to onedimensional spin systems. In order to clarify the properties of spin currents carried by J-W fermions, we focus on the one-dimensional spin-1/2 XXZ model. ${ }^{13,14,15}$ The model reads

$$
\mathcal{H}_{\mathrm{XXZ}}=\Gamma \sum_{i}\left(S_{i}^{x} S_{i+1}^{x}+S_{i}^{y} S_{i+1}^{y}+\Delta_{i} S_{i}^{z} S_{i+1}^{z}\right)
$$

where $S_{l}^{\alpha}$ is $\alpha$ component of a spin- $1 / 2$ operator on $l$ th site $(\alpha=x, y, z)$. The constant parameter $\Gamma$ is the exchange coupling constant, and $\Delta$ is the anisotropic interaction parameter. This model can be well mapped into spinless fermion systems via the J-W transformation. When $\Delta=1$ the XXZ model reduces to the Heisenberg model, and when $\Delta=0$ this model reduces to the XY model; $\mathcal{H}_{\mathrm{XY}}=\Gamma \sum_{i}\left(S_{i}^{x} S_{i+1}^{x}+\right.$ $\left.S_{i}^{y} S_{i+1}^{y}\right)$, which describes the disorder phase; $\left\langle S_{l}^{\alpha}\right\rangle=0$. After a transformation which respects the spin commutation relations; $S_{i}^{x} \rightarrow(-1)^{i} S_{i}^{x}, S_{i}^{y} \rightarrow(-1)^{i} S_{i}^{y}, \quad S_{i}^{z} \rightarrow S_{i}^{z}$, the XXZ model changes the sign of parameters as $\Gamma \rightarrow-\Gamma, \Delta \rightarrow \Delta$. Thus regardless of the signs of the parameters, it is enough to consider the case $\Gamma>0$. This paper focuses on the properties of the spin current when the value of $|\Delta|$ is smaller than one.

Quite recently, theoretical studies for spin currents in one-dimensional spin chains also have been rapidly progressing. Trauzettel et al. ${ }^{16}$ have calculated the ac magnetization current and the power absorption of the XXZ model. Hoogdalem et al. ${ }^{17}$ have considered rectification effects ${ }^{18,19}$ through bosonization techniques in both ferromagnetic and antiferromagnetic systems.

In this paper, we study the effects of external magnetic field gradients along the quantization axis and two-particle interaction gradients on the spin current carried 
by J-W fermions in one-dimensional insulators; the quasiparticle description of onedimensional spin systems is valid in the finite temperature near zero Kelvin. ${ }^{20,21}$ The magnetic field is equivalent to a chemical potential for $\mathrm{J}-\mathrm{W}$ fermions and the gradient plays a role similar to an electric field in electron systems. ${ }^{22}$ The two-particle interaction arises from the anisotropic interaction in eq.(1) and the gradient also generates the spin current. The main aim of this paper is to reveal the temperature dependence of the spin current carried by $\mathrm{J}-\mathrm{W}$ fermions and then compare it with that of the magnon current in the finite temperature near zero Kelvin.

This paper is structured as follows. In the one-dimensional XXZ model, the spin degrees of freedom can be mapped efficiently into fermion degrees of freedom via the $\mathrm{J}-\mathrm{W}$ transformation. Thus first, we define microscopically the spin current density carried by J-W fermions. Second, we evaluate it through the standard procedure of the Schwinger-Keldysh Green's function. The effects of magnetic field gradients and two-particle interaction gradients on the spin current are clarified in Sec.2. Then the magnon current generated by the two-particle interaction (i.e. magnonmagnon interaction) is also calculated in Sec.3. Last, the temperature dependence of the spin current carried by Jordan-Wigner fermions is compared with that of the magnon current in Sec.4. We discuss the cause for the stronger dependence of the spin current carried by $\mathrm{J}-\mathrm{W}$ fermions on temperatures than that of the magnon current in the finite temperature near zero Kelvin.

Table 1. The features of spin currents; spin currents carried by conduction electrons (i.e. conduction electron spin currents), by magnons (i.e. magnon currents) and by J-W fermions (i.e. J-W spin currents). The magnon current, which is the quantized spin-wave spin current, persists for much greater distance than the conduction electron spin current because the dissipation from Joule heating does not exist in insulators.

\begin{tabular}{llll}
\hline Features & Conduction electron spin current & Magnon currents (3-dim) & J-W spin currents (1-dim) \\
\hline - Systems & Metals & Insulators & Insulators \\
- Spins & Conduction electrons' spins & Localized spins & Localized spins \\
- Statistics & Fermions & Bosons & Fermions \\
- Decay length & A few nano-meters & A few centi-meters ${ }^{6}$ & $\backslash$ \\
\hline
\end{tabular}

\section{Jordan-Wigner Spin Current}

The Mermin-Wagner theorem states that continuous symmetries cannot be spontaneously broken at the finite temperature in one- and two-dimensional systems. Therefore in such low-dimensional spin systems, the Holstein-Primakoff transformation is not useful. In other words, because the one-dimensional XXZ model has $\mathrm{SO}(2)$ symmetry that the magnon description cannot be well applied. Thus in onedimensional spin systems, the spin degrees of freedom cannot be mapped efficiently into boson degrees of freedom, but it can be well mapped into spinless fermion degrees of freedom via the $\mathrm{J}-\mathrm{W}$ transformation. Then the $\mathrm{XY}$ model reduces to a free fermion system. 


\subsection{Definition}

The J-W transformation maps the spin operators of the XXZ model onto fermionic operators; $S_{i}^{+}=c_{i}^{\dagger} \Pi_{j=1}^{i-1}\left(1-2 n_{j}\right), S_{i}^{-}=c_{i} \Pi_{j=1}^{i-1}\left(1-2 n_{j}\right), S_{i}^{z}=n_{i}-1 / 2, \quad n_{i} \equiv c_{i}^{\dagger} c_{i}$. Operators $c^{\dagger} / c$ are $\mathrm{J}-\mathrm{W}$ fermion creation/annihilation operators which satisfy the fermionic commutation relation. The operator, $n \equiv c^{\dagger} c$, is the number operator of the J-W fermion. This spinless fermion carries the spin angular momentum of localized spins in one-dimensional systems. The z-component (i.e. $S_{i}^{z}=n_{i}-1 / 2$ ) shows that a spin down can be viewed as an empty site and a spin up corresponds to the presence of a fermion. After the $\mathrm{J}-\mathrm{W}$ transformation and the canonical transformation; $c_{i} \rightarrow(-1)^{i} c_{i}, \mathcal{H}_{\mathrm{XXZ}}$ reads

$$
\mathcal{H}_{\mathrm{XXZ}}=-\frac{\Gamma}{2} \sum_{i}\left(c_{i}^{\dagger} c_{i+1}+c_{i+1}^{\dagger} c_{i}\right)+\Gamma \sum_{i} \Delta_{i}\left(n_{i}-\frac{1}{2}\right)\left(n_{i+1}-\frac{1}{2}\right) .
$$

Eq.(2) shows that only fermions between neighbors feel an interaction $\Gamma \Delta$ and J-W fermions hop between neighboring site with a hopping matrix element $\Gamma / 2$. Therefore in the continuous limit, $\mathcal{H}_{\text {Xxz }}$ reads $\mathcal{H}_{0}+V_{\Delta}$ as an effective Hamiltonian ${ }^{\mathrm{a}}$;

$$
\begin{array}{r}
\mathcal{H}_{0}=\int d x c^{\dagger}(x, t)\left(-\frac{1}{2 m} \frac{d^{2}}{d x^{2}}\right) c(x, t), \\
V_{\Delta}=\int d x J(x) c^{\dagger}(x, t) c^{\dagger}(x, t) c(x, t) c(x, t) .
\end{array}
$$

Here the parameter $m$ is the effective mass of a $\mathrm{J}-\mathrm{W}$ fermion which is related to the curvature of the dispersion relation, and the parameter $J$ corresponds to $-\Gamma \Delta$ in the discrete model.

Moreover, there are magnetic impurities in real materials. Magnetic impurity scatterings make the lifetime of quasiparticles $\tau$, which is inversely proportional to the imaginary part of the self-energy, finite. Here it should be emphasized that the lifetime of quasiparticles in insulators is generally far longer than that of conduction electrons in metals because the dissipation from Joule heating does not exist in insulators; the lifetime of conduction electrons in metals is mainly caused by lattice defects, nonmagnetic impurities and magnetic impurities et al. through Coulomb interactions. Because quasiparticles in insulators have no charge degrees of freedom that the influence of impurities is far smaller than that in metals; magnetic impurities, at most, might cause impurity scatterings. Furthermore in real materials, the rate of impurities such as lattice defects and nonmagnetic impurities is, in general, far larger than that of magnetic impurities. Therefore the imaginary part of the self-energy due to magnetic impurity scatterings can be assumed to be so small that the dispersion relation does not change (i.e. $\omega_{k} \propto k^{2}$ ), and we omit the vertex correction (i.e. the diffusive spin current ${ }^{23}$ ). Let us mention that in eq.(3) we have defined the effective mass of a J-W fermion, $m$, which includes the contribution of the self-energy due to magnetic impurity scatterings.

\footnotetext{
aThrough this paper, we take $\hbar=1$ for convenience.
} 
Thus we introduce the lifetime as a phenomenological parameter and assume that it is independent of temperatures. In order to take account of magnetic impurity scatterings, we adopt retarded and advanced Green's functions, $G_{k, \omega}^{\mathrm{r}}=$ $\left[\omega-\omega_{k}+i /(2 \tau)\right]^{-1}=\left(G_{k, \omega}^{\mathrm{a}}\right)^{*}$ where $\omega_{k}=k^{2} /(2 m)$, on perturbative expansion in Subsec.2.2 and Subsec.2.3. Through these procedure, ${ }^{24}$ the effects of magnetic impurity scatterings, which are the essential dynamics in real materials, are phenomenologically included into our calculation.

The density of J-W fermions, $\rho_{\mathrm{JW}}$, of the system is defined as the expectation value of the number operator of $\mathrm{J}-\mathrm{W}$ fermions as

$$
\rho_{\mathrm{JW}}(x, t) \equiv\left\langle c^{\dagger}(x, t) c(x, t)\right\rangle .
$$

Through Heisenberg's equation of motion, the spin current density carried by J-W fermions, i.e. the $\mathrm{J}-\mathrm{W}$ spin current, $j_{\mathrm{JW}}$, is defined as

$$
\begin{array}{r}
\partial_{t} \rho_{\mathrm{JW}}+\partial_{x} j_{\mathrm{JW}}=0, \\
j_{\mathrm{JW}} \equiv \frac{1}{m} \operatorname{Re}\left[i\left\langle\left(\partial_{x} c^{\dagger}\right) c\right\rangle\right] .
\end{array}
$$

\subsection{Evaluation: magnetic field along the quantization axis}

In this subsection, we consider the $J=0$ case and evaluate the $\mathrm{J}-\mathrm{W}$ spin current generated by magnetic field gradients along the quantization axis. We treat $V_{\mathrm{B}}$; $V_{\mathrm{B}}=-\int d x B(x) S^{z}=-\int d x B(x)\left[c^{\dagger}(x, t) c(x, t)-1 / 2\right]$, as a perturbative term to evaluate the first-order contribution in $B$ without vertex corrections.

Through the standard procedure of the Schwinger-Keldysh (or contour-ordered) Green's function, ${ }^{25,26,27}$ the Langreth method, ${ }^{24,28,29}$ the $\mathrm{J}$-W spin current $j_{\mathrm{JW}}^{\mathrm{B}}$ is evaluated as

$$
\begin{gathered}
j_{\mathrm{JW}}^{\mathrm{B}}=\frac{1}{2 \pi m L^{2}} \operatorname{Im} \sum_{k q}(k+q / 2) \mathrm{e}^{-i q x} B_{-q} \int d \omega \\
\cdot\left(G_{k+q / 2, \omega}^{>} G_{k-q / 2, \omega}^{<}-G_{k+q / 2, \omega}^{\mathrm{t}} G_{k-q / 2, \omega}^{\mathrm{t}}\right)+O\left(B^{2}\right) .
\end{gathered}
$$

The variable $L$ is the chain length and the variables $G^{\mathrm{t}}, G^{>}$, and $G^{<}$are the timeordered, greater, and lesser Green's functions, respectively. Fermionic greater and lesser Green's functions are defined as $G^{>}\left(t_{1}, t_{2}\right) \equiv-i\left\langle c\left(t_{1}\right) c^{\dagger}\left(t_{2}\right)\right\rangle, G^{<}\left(t_{1}, t_{2}\right) \equiv$ $i\left\langle c^{\dagger}\left(t_{2}\right) c\left(t_{1}\right)\right\rangle$. They satisfy the relations: $G_{k, \omega}^{\mathrm{t}}=G_{k, \omega}^{\mathrm{a}}+G_{k, \omega}^{>}, G_{k, \omega}^{>}-G_{k, \omega}^{<}=G_{k, \omega}^{\mathrm{r}}-$ $G_{k, \omega}^{\mathrm{a}}$. Thus the current is rewritten as

$$
\begin{gathered}
j_{\mathrm{JW}}^{\mathrm{B}}=-\frac{1}{2 \pi m L^{2}} \operatorname{Im} \sum_{k q}\left(k+\frac{q}{2}\right) \mathrm{e}^{-i q x} B_{-q} \int d \omega \\
\cdot\left(G_{k+q / 2, \omega}^{\mathrm{a}} G_{k-q / 2, \omega}^{\mathrm{a}}+G_{k+q / 2, \omega}^{\mathrm{a}} G_{k-q / 2, \omega}^{>}+G_{k+q / 2, \omega}^{>} G_{k-q / 2, \omega}^{\mathrm{r}}\right) .
\end{gathered}
$$

The retarded Green's function, $G^{\mathrm{r}}$, is given by $G_{k, \omega}^{\mathrm{r}}=\left[\omega-\omega_{k}+i /(2 \tau)\right]^{-1}=\left(G_{k, \omega}^{\mathrm{a}}\right)^{*}$ and $\tau$ is the lifetime of a J-W fermion. The energy $\omega_{k}$ is $\omega_{k}=F k^{2}, F \equiv(2 m)^{-1}$. Then the function $G^{\mathrm{r}}$ reads $G_{k-q / 2, \omega}^{\mathrm{r}}=G_{k, \omega}^{\mathrm{r}}+\left(G_{k, \omega}^{\mathrm{r}}\right)^{2}\left(-F k q+F q^{2} / 4\right)+O\left((k q)^{2}\right)$. This approximation demands that the magnetic field varies slowly and moderately 
in space (compared to the $\mathrm{J}-\mathrm{W}$ fermion mean-free path), and the value of $\partial_{x} B$ is a constant. Then eq.(9) reads

$$
\begin{aligned}
j_{\mathrm{JW}}^{\mathrm{B}} & =-\frac{4 F \tau}{L^{2}} \operatorname{Im} \sum_{k q}\left(k+\frac{q}{2}\right) \mathrm{e}^{-i q x} i B_{-q} \\
\cdot & \left\{\left[4 \tau\left(1-n_{k}\right)+i \beta \mathrm{e}^{\beta\left(F k^{2}-\mu\right)} n_{k}^{2}\right] F k q\right. \\
- & {\left.\left[4 \tau\left(1-n_{k}\right)-i \beta \mathrm{e}^{\beta\left(F k^{2}-\mu\right)} n_{k}^{2}\right] F k q\right\} } \\
= & \frac{8 F \tau}{L^{2}} \operatorname{Re} \sum_{k} \beta F \mathrm{e}^{\beta\left(F k^{2}-\mu\right)} k^{2} n_{k}^{2} \partial_{x} B
\end{aligned}
$$

where $n_{k}$ is the Fermi distribution function, $\mu$ is the chemical potential, $\beta \equiv 1 /\left(k_{\mathrm{B}} T\right)$ and $k_{\mathrm{B}}$ is the Boltzmann constant. We have used the approximation; $n_{k+q / 2} \approx n_{k}-$ $\beta F \mathrm{e}^{\beta\left(F k^{2}-\mu\right)} n_{k}^{2} k q$. By the Sommerfeld expansion $\left(T \ll T_{\mathrm{F}}, T_{\mathrm{F}} ;\right.$ the Fermi temperature $\left.^{\mathrm{b}}\right)$, the chemical potential is evaluated as $\mu(T)=\epsilon_{\mathrm{F}}+\left(\pi k_{\mathrm{B}} T\right)^{2} /\left(6 \epsilon_{\mathrm{F}}\right)+O\left(T^{4}\right)$, and the summation over $k$ as $\sum_{k} \beta F \mathrm{e}^{\beta\left(F k^{2}-\mu\right)} k^{2} n_{k}^{2} \approx[L /(2 \pi)]\left(\ln 2+\pi^{2} / 24\right) \sqrt{\mu / F}$, where $\epsilon_{\mathrm{F}}$ is the Fermi energy.

Finally, the J-W spin current generated by the magnetic field gradient along the quantization axis in the finite temperature near zero Kelvin reads

$$
j_{\mathrm{JW}}^{\mathrm{B}}=\frac{4 \sqrt{F} \tau}{\pi L}\left(\ln 2+\frac{\pi^{2}}{24}\right) \sqrt{\epsilon_{\mathrm{F}}+\frac{\left(\pi k_{\mathrm{B}} T\right)^{2}}{6 \epsilon_{\mathrm{F}}}} \partial_{x} B .
$$

\subsection{Evaluation: two-particle interaction}

In this subsection, we treat the $B=0$ case and evaluate the $\mathrm{J}-\mathrm{W}$ spin current generated by the two-particle interaction gradient. The twoparticle interaction arises from the anisotropic exchange interaction $V_{\Delta} ; V_{\Delta}=$ $\int d x J(x) c^{\dagger}(x, t) c^{\dagger}(x, t) c(x, t) c(x, t)$, and we treat it as a perturbative term to evaluate the first-order contribution in $J$ without vertex corrections.

Through the same procedure with the last subsection, the J-W spin current generated by the two-particle interaction $V_{\Delta}$ is evaluated as

$$
\begin{gathered}
j_{\mathrm{JW}}^{\Delta}=-\frac{F}{\pi^{2} L^{3}} \operatorname{Im} \sum_{k q}\left(k+\frac{q}{2}\right) \mathrm{e}^{-i q x} J_{-q} i \sum_{\tilde{p}} \int d \omega^{\prime} G_{\tilde{p}, \omega^{\prime}}^{<} \int d \omega \\
\cdot\left(G_{k+q / 2, \omega}^{\mathrm{a}} G_{k-q / 2, \omega}^{\mathrm{a}}+G_{k+q / 2, \omega}^{\mathrm{a}} G_{k-q / 2, \omega}^{>}+G_{k+q / 2, \omega}^{>} G_{k-q / 2, \omega}^{\mathrm{a}}\right. \\
\left.+G_{k+q / 2, \omega}^{>} G_{k-q / 2, \omega}^{>}-G_{k+q / 2, \omega}^{>} G_{k-q / 2, \omega}^{<}\right)+O\left(J^{2}\right) .
\end{gathered}
$$

We have used the relation that the number operator, $\left\langle c^{\dagger}(t) c(t)\right\rangle$, is the same as the equal-time lesser Green's function ${ }^{24,29} ;\left\langle c^{\dagger}(t) c(t)\right\rangle=-i G^{<}(t, t)$. Because each Green's function is not independent; $G^{\mathrm{r}}-G^{\mathrm{a}}=G^{>}-G^{<}$, that eq.(13) is rewritten as

$$
\begin{gathered}
j_{\mathrm{JW}}^{\Delta}=-\frac{F}{\pi^{2} L^{3}} \operatorname{Im} \sum_{k q}\left(k+\frac{q}{2}\right) \mathrm{e}^{-i q x} J_{-q} i \sum_{\tilde{p}} \int d \omega^{\prime} G_{\tilde{p}, \omega^{\prime}}^{<} \int d \omega \\
\cdot\left(G_{k+q / 2, \omega}^{\mathrm{a}} G_{k-q / 2, \omega}^{\mathrm{a}}+G_{k+q / 2, \omega}^{\mathrm{a}} G_{k-q / 2, \omega}^{>}+G_{k+q / 2, \omega}^{>} G_{k-q / 2, \omega}^{\mathrm{r}}\right) .
\end{gathered}
$$

\footnotetext{
${ }^{\mathrm{b}}$ The Fermi temperature of this system(insulator) is estimated as a few hundred Kelvin.
} 
Also in this case, we assume that the two-particle interaction varies slowly and moderately in space (compared to the J-W fermion mean-free path), and the value of $\partial_{x} J$ is a constant. Therefore the retarded green's function can be approximated; $G_{k-q / 2, \omega}^{\mathrm{r}}=G_{k, \omega}^{\mathrm{r}}+\left(G_{k, \omega}^{\mathrm{r}}\right)^{2}\left(-F k q+F q^{2} / 4\right)+O\left((k q)^{2}\right)$. Then eq.(14) reads

$$
j_{\mathrm{JW}}^{\Delta}=-\frac{16 F \tau}{L^{3}} \operatorname{Re} \sum_{k} \beta F \mathrm{e}^{\beta\left(F k^{2}-\mu\right)} k^{2} n_{k}^{2} \sum_{\tilde{p}} n_{\tilde{p}} \partial_{x} J .
$$

The summation over $\tilde{p}$ is evaluated as $\sum_{\tilde{p}} n_{\tilde{p}} \approx[L /(2 \pi \sqrt{F})][2 \sqrt{\mu}-$ $\left.\left(\pi k_{\mathrm{B}} T\right)^{2} /\left(12 \mu^{3 / 2}\right)\right]$. Finally, the J-W spin current generated by the two-particle interaction in the finite temperature near zero Kelvin reads

$$
j_{\mathrm{JW}}^{\Delta}=-\frac{4 \tau}{\pi^{2} L}\left(\ln 2+\frac{\pi^{2}}{24}\right)\left\{2\left[\epsilon_{\mathrm{F}}+\frac{\left(\pi k_{\mathrm{B}} T\right)^{2}}{6 \epsilon_{\mathrm{F}}}\right]-\frac{\left(\pi k_{\mathrm{B}} T\right)^{2}}{12\left[\epsilon_{\mathrm{F}}+\frac{\left(\pi k_{\mathrm{B}} T\right)^{2}}{6 \epsilon_{\mathrm{F}}}\right]}\right\} \partial_{x} J
$$

\section{Magnon Current}

According to the Mermin-Wagner theorem, three-dimensional spin systems are the most suitable set-ups for the study of magnon transports. Therefore in order to clarify the thermal properties of magnon currents, we focus on three dimensional ferromagnetic insulators at the low-temperature near zero Kelvin, which has no conduction electrons. The spin degree of freedom reduces to the magnon degree of freedom via the Holstein-Primakoff transformation; the transverse components of the exchange interactions give rise to hopping of the magnons, while the longitudinal component give rise to the interaction.

External magnetic fields, of course, generate magnon currents also in this case; the magnetic field along the quantization axis is equivalent to a chemical potential for magnons as well as J-W fermions, and the gradient generates magnon currents. Moreover time-dependent transverse magnetic fields cause quantum fluctuations and generate a magnon current through pumping effects. ${ }^{30}$ Here, however, we consider three dimensional ferromagnetic insulators without any external magnetic fields.

\subsection{Definition}

The low-energy effective Hamiltonian ${ }^{31}$ for magnons in three-dimensional insulators reads $\mathcal{H}_{\mathrm{m}}=\mathcal{H}_{0(\mathrm{~m})}+V_{\mathrm{m}}$;

$$
\begin{gathered}
\mathcal{H}_{0(\mathrm{~m})}=\int d^{3} x a^{\dagger}(\mathbf{x}, t)\left(-\frac{\nabla^{2}}{2 m_{\mathrm{mag}}}\right) a(\mathbf{x}, t), \\
V_{\mathrm{m}}=\int d^{3} x J_{(\mathrm{m})}(\mathbf{x}) a^{\dagger}(\mathbf{x}, t) a^{\dagger}(\mathbf{x}, t) a(\mathbf{x}, t) a(\mathbf{x}, t) .
\end{gathered}
$$


Here $m_{\text {mag }}$ is the effective mass of a magnon, and $V_{\mathrm{m}}$ represents the magnon-magnon interaction. Operators $a^{\dagger} / a$ are magnon creation/annihilation operators which satisfy the bosonic commutation relation. In the low-temperature, only the two-particle interaction $J_{(\mathrm{m})}$ is important. ${ }^{32,33}$

The magnetic impurity scattering is, of course, an inevitable dynamics in real materials even when the system is under low-temperature. It makes the lifetime of magnons, $\tau_{\mathrm{m}}$, finite. Therefore by the same procedure with Subsec.2.1, we phenomenologically include the effects of magnetic impurity scatterings, which are the inevitable dynamics in real materials, into our calculation. Also in this case, we have assumed that the imaginary part of the self-energy due to magnetic impurity scatterings is so small that the dispersion relation does not change, and we omit the vertex correction (i.e. the diffusive spin current). Furthermore, we assume that the lifetime of magnons is independent of temperatures.

The magnon density, $\rho_{\mathrm{m}}$, of the system is defined as the expectation value of the number operator of magnons

$$
\rho_{\mathrm{m}}(\mathbf{x}, t) \equiv\left\langle a^{\dagger}(\mathbf{x}, t) a(\mathbf{x}, t)\right\rangle
$$

Through Heisenberg's equation of motion, the magnon current (density), $\mathbf{j}_{\mathrm{m}}$, is defined as

$$
\frac{\partial \rho_{\mathrm{m}}}{\partial t}+\nabla \cdot \mathbf{j}_{\mathrm{m}}=0
$$

Then the magnon current reads

$$
j_{\mathrm{m}}^{\nu}(\mathbf{x}, t)=\frac{1}{m_{\mathrm{mag}}} \operatorname{Re}\left[i\left\langle\left(\partial_{\nu} a^{\dagger}(\mathbf{x}, t)\right) a(\mathbf{x}, t)\right\rangle\right],
$$

where $\nu$ is a direction for a magnon current to flow $(\nu=x, y, z)$.

\subsection{Evaluation}

From now on, we treat the magnon-magnon interaction term, $V_{\mathrm{m}}$, as a perturbative one to evaluate the first-order contribution in $J_{(\mathrm{m})}$ without vertex corrections. Through the same procedure with the last section, the Langreth method, ${ }^{24,28,29}$ the magnon current is evaluated as

$$
\begin{gathered}
j_{\mathrm{m}}^{\nu}=-\frac{4}{m_{\mathrm{mag}}}\left(\frac{2 \pi}{V}\right)^{3} \operatorname{Re} \sum_{\mathbf{k}, \mathbf{q}}\left(k_{\nu}+\frac{q_{\nu}}{2}\right) \mathrm{e}^{-i \mathbf{q} \cdot \mathbf{x}} J_{(\mathrm{m})-\mathbf{q}} \sum_{\tilde{\mathbf{p}}} \int \frac{d \omega^{\prime}}{2 \pi} \tilde{G}_{\tilde{\mathbf{p}}, \omega^{\prime}}^{<} \int \frac{d \omega}{2 \pi} \\
\cdot\left(\tilde{G}_{\mathbf{k}+\mathbf{q} / 2, \omega}^{\mathrm{a}} \tilde{G}_{\mathbf{k}-\mathbf{q} / 2, \omega}^{\mathrm{a}}+\tilde{G}_{\mathbf{k}+\mathbf{q} / 2, \omega}^{\mathrm{a}} \tilde{G}_{\mathbf{k}-\mathbf{q} / 2, \omega}^{>}+\tilde{G}_{\mathbf{k}+\mathbf{q} / 2, \omega}^{>} \tilde{G}_{\mathbf{k}-\mathbf{q} / 2, \omega}^{\mathrm{r}}\right)+O\left(J_{(\mathrm{m})}^{2}\right)(22)
\end{gathered}
$$

Here $V$ is a volume of the system. Bosonic greater and lesser Green's functions are defined as $\tilde{G}^{>}\left(t_{1}, t_{2}\right) \equiv-i\left\langle a\left(t_{1}\right) a^{\dagger}\left(t_{2}\right)\right\rangle, \tilde{G}^{<}\left(t_{1}, t_{2}\right) \equiv-i\left\langle a^{\dagger}\left(t_{2}\right) a\left(t_{1}\right)\right\rangle$; the sign of the lesser green's function is different from the fermionic case. The retarded Green's function is $\tilde{G}_{\mathbf{k}, \omega}^{\mathrm{r}}=\left[\omega-\tilde{\omega}_{\mathbf{k}}+i /\left(2 \tau_{\mathrm{m}}\right)\right]^{-1}=\left(\tilde{G}_{\mathbf{k}, \omega}^{\mathrm{a}}\right)^{*}$, where the energy $\tilde{\omega}_{\mathbf{k}}$ is $\tilde{\omega}_{\mathbf{k}}=D k^{2}$, $D \equiv 1 /\left(2 m_{\mathrm{mag}}\right)$ and $\tau_{\mathrm{m}}$ is the lifetime of magnons. The lifetime represents the damping of spins $\left(\tau_{\mathrm{m}}\right.$ is inversely proportional to the Gilbert damping parameter, $\alpha)$. Then the retarded Green's function satisfies $\tilde{G}_{\mathbf{k}+\mathbf{q} / 2, \omega}^{\mathrm{r}}=\tilde{G}_{\mathbf{k}, \omega}^{\mathrm{r}}+\left(\tilde{G}_{\mathbf{k}, \omega}^{\mathrm{r}}\right)^{2}(D \mathbf{k}$. 
$\left.\mathbf{q}+q^{2} / 4\right)+O\left((\mathbf{k} \cdot \mathbf{q})^{2}\right)$. This approximation demands that the magnon-magnon interaction varies slowly and moderately (compared to the magnon mean-free path), and the value of $\partial_{\nu} J_{(\mathrm{m})}$ is a constant.

Thus eq.(22) is rewritten as

$$
\begin{aligned}
j_{\mathrm{m}}^{\nu}=\frac{8 D^{2} \tau_{\mathrm{m}}}{V^{2}} & \operatorname{Re} \sum_{\mathbf{k}, \mathbf{q}}\left(k_{\nu}+\frac{q_{\nu}}{2}\right) \mathrm{e}^{-i \mathbf{q} \cdot \mathbf{x}} J_{(\mathrm{m})-\mathbf{q}} i \zeta\left(\frac{3}{2}\right) \Gamma\left(\frac{3}{2}\right)\left(\frac{k_{\mathrm{B}} T}{D}\right)^{3 / 2} \\
& \cdot\left\{\left[\beta\left(\mathrm{e}^{-\beta D k^{2}}+2 \mathrm{e}^{-2 \beta D k^{2}}\right)+4 i \tau_{\mathrm{m}}\left(1+\tilde{n}_{\mathbf{k}}\right)\right] \mathbf{k} \cdot \mathbf{q}\right. \\
+ & {\left.\left[\beta\left(\mathrm{e}^{-\beta D k^{2}}+2 \mathrm{e}^{-2 \beta D k^{2}}\right)-4 i \tau_{\mathrm{m}}\left(1+\tilde{n}_{\mathbf{k}}\right)\right] \mathbf{k} \cdot \mathbf{q}\right\} } \\
=\frac{16 \tau_{\mathrm{m}}}{3 V^{2}} \zeta\left(\frac{3}{2}\right) \Gamma\left(\frac{3}{2}\right) & \sqrt{D k_{\mathrm{B}} T} \operatorname{Re} \sum_{\mathbf{k}} k^{2}\left(\mathrm{e}^{-\beta D k^{2}}+2 \mathrm{e}^{-2 \beta D k^{2}}\right) \sum_{\mathbf{q}} i q_{\nu} \mathrm{e}^{-i \mathbf{q} \cdot \mathbf{x}} J_{(\mathrm{m})-\mathbf{q}_{\mathbf{2}}}(23)
\end{aligned}
$$

Here $\tilde{n}_{\mathbf{k}}$ is the Bose distribution function, $\zeta$ is the Riemann zeta function, $\zeta(3 / 2)=$ 2.612 , and $\Gamma$ is the Euler gamma function, $\Gamma(3 / 2)=\sqrt{\pi} / 2$. Because we consider the the low-temperature regime near zero Kelvin, we have approximated as $\tilde{n}_{\mathbf{k}+\mathbf{q} / 2} \approx$ $\tilde{n}_{\mathbf{k}}-\beta D \mathrm{e}^{-\beta D k^{2}}\left(1-\mathrm{e}^{-\beta D k^{2}}\right)^{-2} \mathbf{k} \cdot \mathbf{q} \approx \tilde{n}_{\mathbf{k}}-\beta D\left(\mathrm{e}^{-\beta D k^{2}}+2 \mathrm{e}^{-2 \beta D k^{2}}\right) \mathbf{k} \cdot \mathbf{q}$. Moreover we have used the isotropy condition in the direction for $\mathbf{k} ; \sum_{\mathbf{k}} k_{\nu} \mathbf{k} \cdot \mathbf{q}=\sum_{\mathbf{k}} k^{2} q_{\nu} / 3$.

Finally in the low-temperature near zero Kelvin, the magnon current generated by the magnon-magnon interaction gradient reads

$$
j_{\mathrm{m}}^{\nu}=-\frac{D \tau_{\mathrm{m}}}{V}\left(1+\frac{1}{2^{3 / 2}}\right) \zeta\left(\frac{3}{2}\right) \Gamma\left(\frac{3}{2}\right)\left(\frac{k_{\mathrm{B}} T}{D \sqrt{\pi}}\right)^{3} \partial_{\nu} J_{(\mathrm{m})} .
$$

Table 2. The temperature dependence of J-W spin currents and magnon ones generated by the magnetic field gradient and by the two-particle interaction gradient. The constant $\gamma$ is defined as $\gamma \equiv 6\left[\epsilon_{\mathrm{F}} /\left(\pi k_{\mathrm{B}}\right)\right]^{2} . \mathrm{J}-\mathrm{W}$ spin currents exhibit the stronger dependence on temperatures than magnon ones in the finite temperature near zero Kelvin (i.e. $0<T<1[\mathrm{~K}]$ ).

\begin{tabular}{lll}
\hline Temperature dependence & J-W spin currents (1-dim) & Magnon currents (3-dim) \\
\hline - Magnetic field; $\partial_{\nu} B$ & $\propto \sqrt{\gamma+T^{2}}$ & $\propto T^{3 / 2}($ Ref.6) \\
- Two-particle interaction; $\partial_{x} J,\left(\partial_{\nu} J_{(\mathrm{m})}\right)$ & $\propto\left[\gamma+T^{2}-T^{2} /\left(4+4 T^{2} / \gamma\right)\right]$ & $\propto T^{3}$ \\
\hline
\end{tabular}

\section{Temperature Dependence near Zero Kelvin}

The Mermin-Wagner theorem gives crucial differences between low-dimensional spin systems and three-dimensional ones. It prohibits the spontaneous breaking of the continuous symmetry of the low-dimensional XXZ model, i.e. SO(2) symmetry, in the finite temperature. In other words, in low-dimensional systems quantum fluctuations are so strong as to destroy magnetic orders. Then the thermal properties of each spin current in insulators, the $\mathrm{J}-\mathrm{W}$ spin current and the magnon one, are drastically different from each other in the finite temperature near zero Kelvin (i.e. $0<T<1[\mathrm{~K}]$ ); as shown in Table 2, the J-W spin current exhibits the stronger dependence on temperatures than the magnon current, i.e. the exponents of 
temperatures in J-W spin currents are smaller than those in magnon currents. Thus, the influence of quantum and thermal fluctuations in one-dimensional insulators is larger than that in three-dimensional ones. The Table 2 also shows that the current generated by the two-particle interaction gradient shows the weaker dependence on temperatures than the one by the magnetic field gradient along the quantization axis.

\section{Summary and Discussion}

We have theoretically studied the temperature dependence of the spin current in one- and three-dimensional insulators. In one-dimensional insulators, the spin current is carried by Jordan-Wigner fermions. In this system, quantum fluctuations are strong and the J-W spin current shows the stronger dependence on temperatures than the magnon one in the finite temperature near zero Kelvin.

Experimentally though the direct measurement of spin currents is impossible at this stage, the spin currents we have discussed would be identified by observing the temperature dependence of the spin currents through inverse spin-Hall effects. Theoretically, the microscopic calculation of the lifetime caused by magnetic impurity scatterings and also by phonon ones et al., and the diffusive spin current with vertex corrections is an significant theoretical issue.

\section{Acknowledgements}

The author would like to thank K.Totsuka, G.Tatara, S.Fujimoto and M.Oshikawa. He is also grateful to Y.Korai for invaluable discussion and T.Kimura for reading of the manuscript and useful comments. We wish to acknowledge the G.Tataralaboratory members in Tokyo Metropolitan University for warm hospitality during his stay.

This work was supported by the Grant-in-Aid for the Global COE Program "The Next Generation of Physics, Spun from Universality and Emergence" from the Ministry of Education, Culture, Sports, Science and Technology (MEXT) of Japan.

\section{References}

1. S.Maekawa, Concepts in Spin Electronics (Oxford science publications, 2006).

2. Y.Tserkovnyak, A.Brataas, G.E.W.Bauer, and B.I.Halperin, Rev.Mod.Phys. 77, 1375 (2005).

3. D.D.Awschalom and M.E.Flatte, Nature Phys 3, 153 (2007).

4. S. A. Wolf, D. D. Awschalom, R. A. Buhrman, J. M. Daughton, S. von Molnar, M. L. Roukes, A. Y. Chtchelkanova, and D. M. Treger, Science 294, 1488 (2001).

5. I.Zutic, J.Fabian, and S.D.Sarma, Rev.Mod.Phys. 76, 323 (2004).

6. Y.Kajiwara, K.Harii, S.Takahashi, J.Ohe, K.Uchida, M.Mizuguchi, H.Umezawa, H.Kawai, K.Ando, K.Takanashi, S.Maekawa, and E.Saitoh, Nature 464, 262 (2010).

7. Y.K.Kato, R.C.Myers, A.C.Gossard, and D.D.Awschalom, Science 306, 1910 (2004). 
8. F.Meier and D.Loss, Phys.Rev.Lett. 90, 167204 (2003).

9. S.Fujimoto, Phys.Rev.Lett. 103, 047203 (2009).

10. H.Katsura, N.Nagaosa, and P.A.Lee, Phys.Rev.Lett. 104, 066403 (2010).

11. Y.Onose, T.Ideue, H.Katsura, Y.Shiomi. N.Nagaosa, and Y.Tokura, Science 329, 297 (2010).

12. R.Matsumoto and S.Murakami, Phys.Rev.Lett. 106, 197202 (2011).

13. E.Fradkin, Field Theories of Condensed Matter Systems (Allan M.Wylde,1991).

14. I. Affleck, in Fields, Strings and Critical Phenomena, Les Houches Summer School Proceedings, Vol 49 ( Elsevier Science, Amsterdam, 1989).

15. T.Giamarchi, Quantum Physics in One Dimension ( Oxford University Press, 2003).

16. B.Trauzettel, P.Simon, and D.Loss, Phys.Rev.Lett. 101, 017202 (2008).

17. K.A.van. Hoogdalem and D.Loss, "Rectification of spin currents in spin chains", condmat/11024801.

18. D.E.Feldman, S.Scheidl, and V.M.Vinokur, Phys.Rev.Lett. 94, 186809 (2005).

19. B.Braunecker, D.E.Feldman, and F.Li, Phys.Rev.B 76, 085119 (2007).

20. A.Virosztek and J.Ruvalds, Phys.Rev.B 42, 4064 (1990).

21. S.Fujimoto, Bussei Kenkyu. 54, 207 (1990).

22. F.D.M.Haldane and D.P.Arovas, Phys.Rev.B 52, 4223 (1995).

23. A.Takeuchi, K.Hosono, and G.Tatara, Phys.Rev.B 81, 144405 (2010).

24. H. Haug and A.P. Jauho, Quantum Kinetics in Transport and Optics of Semiconductors (Springer New York, 2007).

25. J.Rammer and H.Smith, Rev.Mod.Phys. 58, 323 (1986).

26. A.Kamenev, "Many-body theory of non-equilibrium systems", cond-mat/0412296.

27. T.Kita, Prog.Theor.Phys. 123, 581 (2010).

28. G.Tatara, H.Kohno, and J.Shibata, Physics Report. 468, 213 (2008).

29. D. A. Ryndyk, R. Gutierrez, B. Song and G. Cuniberti, Energy Transfer Dynamics in Biomaterial Systems, (Springer-Verlag, 2009), pp. 213-335.

30. K.Nakata and G.Tatara, J. Phys. Soc. Jpn. 80, 054602 (2011).

31. T.Nikuni, M.Oshikawa, A.Oosawa, and H.Tanaka, Phys.Rev.Lett. 84, 5868 (2000).

32. A.K.Kolezhuk, Phys.Rev.B 59, 4181 (1999).

33. T.Giamarchi and A.M.Tsvelik, Phys.Rev.B 59, 11398 (1999). 